\title{
On the weakness of linear programming to interpret the nature of solution of fully fuzzy linear system
}

\author{
Ghassan Malkawi ${ }^{1,2^{*}}$, Nazihah Ahmad ${ }^{1}$ and Haslinda Ibrahim ${ }^{1}$
}

\author{
* Correspondence: \\ gassan_malkawi@yahoo.com \\ ${ }^{1}$ College of Arts and Sciences, \\ University Utara Malaysia, 06010 \\ Sintok, Kedah, Malaysia \\ ${ }^{2}$ Mathematics Department, College \\ of Arts and Sciences, Northern \\ Borders University, Rafha, Kingdom \\ of Saudi Arabia
}

\begin{abstract}
One of the applications of linear programing is to get solutions for fully fuzzy linear system (FFLS) when the near-zero fuzzy number is considered. This usage could be applied to interpret the nature of FFLS solution according to the nature of FFLS solution in the work of Babbar et al. (Soft Comput. 17:1-12, 2012) and Kumar et al. (Advances in Fuzzy Systems 2011:1-8, 2011). This paper shows that the nature of FFLS solutions must not depend upon the nature of linear programming (LP) solutions, because LP is not enough to obtain all the exact solutions for FFLS which contradicts the claims of researchers. Counter examples are provided in order to falsify those claims. Numerically, we confirm that the nature of the possible way of solving FFLS is completely different from that of the linear system. For instance, FFLS may have two unique solutions which contradict the uniqueness that can be obtained through only one unique solution.
\end{abstract}

Keywords: Fully fuzzy linear system; Fuzzy number; Near-zero fuzzy number; Linear programing

\section{Introduction}

Linear system of equations is the simplest framework and the most beneficial mathematical model for many problems considered by applied mathematics. In practice, unfortunately, the accurate values of coefficients of these systems are not available and not well defined in many applications. This uncertainty may either be probabilistic or nonprobabilistic in nature. Accordingly, mathematical tools were developed to represent and deal with vagueness and fuzziness. The response is the fuzzy theory, which provides a widely appreciated tool and representation of these uncertain data in many fields.

Zadeh was the pioneer in the field of fuzzy sets and systems in [1,2]. The application of this theory, in linear system of equations where the elements of the matrix are crisp numbers and the elements of vector are fuzzy numbers, leads to a new system called fuzzy linear system (FLS). On the other hand, it is called fully fuzzy linear system (FFLS), when the all elements are fuzzy numbers. The problem for solving the fuzzy systems is that, we must use methods without using inverse operators, because the extended operation on fuzzy numbers, subtraction and division of fuzzy numbers are not the inverse operations to addition and multiplication, respectively [3,4].

\section{Springer}

(c) 2014 Malkawi et al.; licensee Springer. This is an Open Access article distributed under the terms of the Creative Commons Attribution License (http://creativecommons.org/licenses/by/2.0), which permits unrestricted use, distribution, and reproduction in any medium, provided the original work is properly credited. 
Buckley and $\mathrm{Qu}$ in $[5,6]$ discussed the theoretical features of the existence of a solution of fuzzy systems, they provided categories of solution for FFLS as the classical solution $X_{C}$, the vector solution $X_{J}$, and the marginal solutions $X_{E}$ and $X_{I}$. Their methods were generalized in [7] to a fuzzy system of equations $A_{1} x+b_{1}=A_{2} x+b_{2}$, where $A_{1}$, $A_{2}, b_{1}$, and $b_{2}$ are fuzzy matrices of fuzzy numbers.

The first achievable approach of FLS was obtained in [8], where they proposed a generic model for solving an $n \times n$ FLS by employing the embedding approach. The most available study of FFLS was obtained in [9]; they studied a special case, where all coefficients and parameters are positives. In their study, they declared that there are infinite numbers of scenarios and states that can be constructed from an FFLS. The methods of finding a solution for these scenarios of FFLS create new scenarios of fuzzy system which are based on these actual scenarios of FFLS [10].

Dehghan and his colleagues in [11-13] found the solution for FFLS where the coefficient and parameters are positive; their methods were based on the positivity case in the approximate arithmetic operators on LR fuzzy numbers in [14]. Furthermore, scholars in [15-18] and [19-23] proposed new methods for solving FFLS in a similar case to Dehghan and his colleagues. In [24-27], researchers generalized methods for solving FFLS where the coefficients and the parameters are not only positive, but they also restrict the signs of coefficient or parameters only to positive or negative in one side of FFLS in an attempt to avoid the near-zero triangular fuzzy numbers in both sides of FFLS. In [28], Kumar et al. obtained an exact and infinite positive solution for positive FFLS; moreover, they employed a similar technique to find a positive solution for negative FFLS in [29]. In [30], Malkawi et al. proposed new matrix methods for solving a positive FFLS, the necessary and sufficient condition to have a positive solution was discussed, and their methods and results were also capable of solving left-right fuzzy linear system (LR-FLS) and FLS.

In [31], they found approximate solution for FFLS; the near-zero fuzzy numbers are not included. They proposed a numerical method founded by a fuzzy neural network denoted by FNN. The method was restricted by many constraints. The method can solve only when the system has a unique fuzzy solution and the matrix has to be squares which prevent extension of the method to solve a rectangle matrix $\tilde{A}$. To overcome the shortcomings in previous methods, the researchers relied heavily on linear programming (LP) to propose methods that can consider the near-zero triangular fuzzy numbers in both sides of FFLS in $[32,33]$. The authors in the two studies declared that the nature of the solutions of the FFLS depends on the nature of the solutions of the LP, i.e., the possible way of solution for FFLS may not be a single unique solution but infinitely many solutions.

Unfortunately, LP can give answers to the linear system but not in FLS and FFLS. The LP technique cannot obtain all feasible solutions for FLS and FFLS. Kumar and his colleagues illustrated examples of one solution while it has two unique fuzzy solutions or infinite number of solutions as will be illustrated through examples in this paper. Also, they added many restrictions to the systems. These restrictions required many steps and longer time to reach the ultimate solution; moreover, their examples did not exceed fuzzy matrix $\tilde{A}$ of size $n=2$. However, all examples in the literature do not exceed fuzzy matrix $\tilde{A}$ of size $2 \times 3$, if the near-zero LR fuzzy number appears in both hand sides for FFLS. 
In this paper, we show that the nature of the solutions of the FFLS is completely different from the nature of the solutions of the LP and that it is insufficient to obtain all exact solutions for FFLS, using numerical examples. We employed the results from this survey as examples for LP methods in specific studies in [32,33] to declare that the consistency or possible solution for FFLS is not similar to the known consistency concept in the linear system. For instance, the FFLS may yield two unique solutions or many infinite solutions despite that it is constructed by only one equation. Also, the number of solutions in non-square FFLS does not depend on the number of equations compared with the number of parameters.

The structure of this paper is organized as follows: in the 'Preliminaries' section, the basic definitions of the fuzzy set theory are provided. In the 'Fully fuzzy linear system' section, we intend to illustrate the concept of FFLS, and hence, a brief summary on solving the FFLS using LP technique is provided. The dissection is contained in the 'Numerical examples' section where the problems in LP technique are pointed out numerically. In this section, many examples are solved by other methods in the literature or directly by an associated system; however, the verifications of numerical examples are provided. Since the verification of the solutions may be obtained also using distance metric function [34], some examples are verified by distance metric function. We provide the final result without considering the used methods because the aim of this paper is to show the weakness of the LP method to detect all exact feasible solutions, and we want to confirm that the nature of solution for LP which has been gained by optimal solution cannot explain the nature of the solution for FFLS. In the 'Conclusion' section, we conclude the paper.

\section{Preliminaries}

In this section, basic definitions and notions of fuzzy set theory are reviewed $([14,35,36])$.

Definition 1. Let $X$ be a universal set. Then, we define the fuzzy subset $\tilde{A}$ of $X$ by its membership function $\mu_{\tilde{A}}(x): \mathbb{R} \rightarrow[0 ; 1]$ which assigns to each element $x \in X$ a real number $\mu_{\tilde{A}}(x)$ in the interval $[0,1]$, where the value $\mu_{\tilde{A}}(x)$ represents the grade of membership of $x$ in $\tilde{A}$.

A fuzzy set $A$ is written as $\tilde{A}=\left\{\left(x, \mu_{\tilde{A}}(x)\right), x \in X, \mu_{\tilde{A}}(x) \in[0,1]\right\}$.

Definition 2. A fuzzy set $\tilde{A}$ in $X=\mathbb{R}^{\mathrm{n}}$ is convex fuzzy set if

$$
\begin{aligned}
& \forall x_{1}, x_{2} \in X, \forall \lambda \in[0,1], \\
& \mu_{\tilde{A}}\left(\lambda x_{1}+(1-\lambda) x_{2}\right) \geq \min \left(\mu_{\tilde{A}}\left(x_{1}\right), \mu_{\tilde{A}}\left(x_{2}\right)\right) .
\end{aligned}
$$

Definition 3. Let $\tilde{A}$ be a fuzzy set defined on the set of real numbers $\mathbb{R}$. $\tilde{A}$ is called normal fuzzy set if there exists $x \in \mathbb{R}$ such that $\mu_{\tilde{A}}(x)=1$.

Definition 4. A fuzzy number is a normal and convex fuzzy set, with its membership function $\mu_{\tilde{A}}(x)$ defined in real line $\mathbb{R}$ and piecewise continuous.

Definition 5. (Left-right fuzzy number) A fuzzy number $\tilde{m}$ is called left-right fuzzy number, abbreviated as LR fuzzy number, where its membership function satisfies 


$$
\mu_{\tilde{m}}(x)=\left\{\begin{array}{c}
L\left(\frac{m-x}{\alpha}\right), \text { for } x \leq m, \alpha>0, \\
R\left(\frac{x-m}{\beta}\right), \text { for } m \leq x, \beta>0,
\end{array}\right.
$$

where $m, \alpha, \beta \in \mathbb{R}$

and the function $L($.$) is called a left shape function if the following hold:$

1. $L(x)=L(-x)$

2. $L(0)=1, L(1)=0$

3. $L$ is non-increasing on $[0, \infty]$

Also, the definition of function $R($.$) which called right shape is similar to that of L($.$) .$ It is symbolically written as $\tilde{m}=(m, \alpha, \beta)_{\mathrm{LR}}$, where $m$ symbolizes the mean value, while $\alpha$ and $\beta$ are left and right spreads, respectively. We denote the set of LR fuzzy numbers $F(\Re)$.

The sign of $\tilde{m}=(m, \alpha, \beta)_{\mathrm{LR}}$ is classified as follows:

- $\tilde{m}$ is called positive (negative) iff $m-\alpha \geq 0(\beta+m \leq 0)$.

- $\tilde{m}$ is called zero if $(m=0, \alpha, \beta=0)$.

- $\tilde{m}$ is called near zero iff $m-\alpha<0<\beta+m$.

Definition 6. Two fuzzy numbers $\tilde{n}=(n, \gamma, \delta)_{\mathrm{LR}}$ and $\tilde{m}=(m, \alpha, \beta)_{\mathrm{LR}}$ are called equal, iff $(n=m, \gamma=\alpha, \delta=\beta)$.

Definition 7. (Arithmetic operations on LR fuzzy numbers) We will represent arithmetic operations for two LR fuzzy numbers $\tilde{m}=(m, \alpha, \beta)_{\mathrm{LR}}$ and $\tilde{n}=(n, \gamma, \delta)_{\mathrm{LR}}$ as follows:

- Addition:

$$
(m, \alpha, \beta)_{\mathrm{LR}} \oplus(n, \gamma, \delta)_{\mathrm{LR}}=(m+n, \alpha+\gamma, \beta+\delta)_{\mathrm{LR}}
$$

- Opposite:

$-(m, \alpha, \beta)_{\mathrm{LR}}=-(m, \alpha, \beta)_{\mathrm{LR}}=(-m, \beta, \alpha)_{\mathrm{RL}}$

- Subtraction:

$$
(m, \alpha, \beta)_{\mathrm{LR}} \ominus(n, \gamma, \delta)_{\mathrm{RL}}=(m-n, \alpha+\delta, \beta+\gamma)_{\mathrm{LR}}
$$

Definition 8. A popular LR fuzzy number is a triangular fuzzy number (abbreviated TFN), where $L=R=\max (0,1-x)$; consequently, using (1), its membership function is given by 


$$
\mu_{\tilde{A}}(x)=\left\{\begin{array}{c}
1-\frac{m-x}{\alpha}, m-\alpha \leq x<m, \alpha>0, \\
1-\frac{x-m}{\beta}, m \leq x<m+\beta, \beta>0 . \\
0 \text { otherwise. }
\end{array}\right.
$$

It is symbolically written as a triangular fuzzy number $\tilde{m}=(m, \alpha, \beta)$.

Example 1. The membership function of the near-zero triangular fuzzy number $\tilde{m}=$ $(3,5,4)$ (Figure 1) is

$$
\mu_{\tilde{m}}(x)=\left\{\begin{array}{cc}
0, & x<-2, \\
\frac{2+x}{5}, & -2 \leq x \leq 3, \\
\frac{7-x}{4}, & 3 \leq x \leq 7, \\
0, & \text { otherwise. }
\end{array}\right.
$$

Note 1. The triangular fuzzy number can be represented in another form; it is derived if we suppose

$$
a=m-\alpha, b=m, c=m+\beta .
$$

In this case, it is symbolically written as $\tilde{a}^{\prime}=\left(a_{1}, a_{2}, a_{3}\right)$, or $\tilde{a}^{\prime}=(a, b, c)$.

Then, the membership function for this form is

$$
\mu_{\tilde{a}}(x)=\left\{\begin{array}{cc}
\frac{x-a}{b-a}, & a \leq x \leq b, \\
\frac{c-x}{c-b}, & b \leq x \leq c, \\
0, & \text { otherwise. }
\end{array}\right.
$$

The TFN for $\tilde{m}=(3,5,4)$ in Example 1 is written from $(a, b, c)$ as

$$
\tilde{m}^{\prime}=(3-5,3,3+5)=(-2,3,7) \text {. }
$$

In this paper, we used triangular fuzzy number (TFN) in the form $(m, \alpha, \beta)$, and when a method used the other form $(a, b, c)$, the example rewrites in two forms.

Definition 9. (Kaufmann's approximation for multiplication of TFN) Let $\tilde{m}=(m, \alpha, \beta)$ and $\tilde{n}=(n, \gamma, \delta)$ be two unrestricted triangular fuzzy numbers

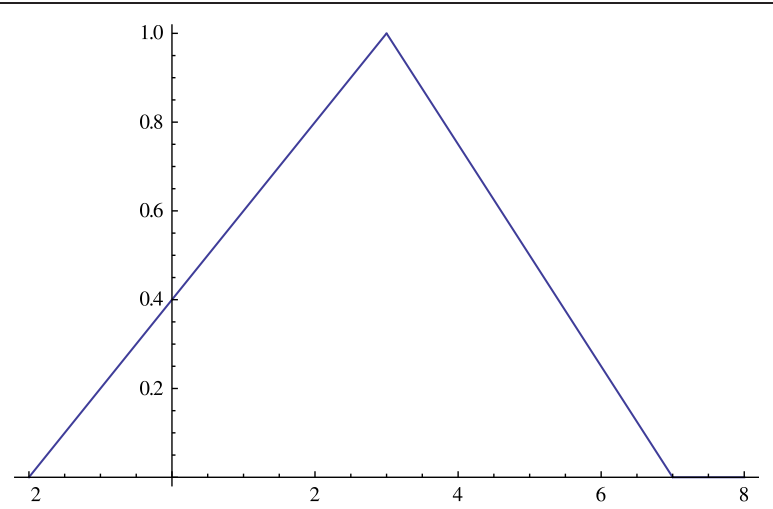

Figure 1 Triangular fuzzy number $m=(3,5,4)$. 


$$
\tilde{m} \otimes \tilde{n}=\left(m n, f_{1}, f_{2}\right)
$$

Where

$$
\begin{gathered}
f_{1}=m n-\min \{(m-\alpha)(n-\gamma),(m+\beta)(n-\gamma),(m+\beta)(n+\delta), \\
(m-\alpha)(n+\delta)\},
\end{gathered}
$$

and

$$
f_{2}=\max \{(m-\alpha)(n-\gamma),(m+\beta)(n-\gamma),(m+\beta)(n+\delta),(m-\alpha)(n+\delta)\}-m n .
$$

Definition 10. A vector $\tilde{X}=\left(\tilde{x}_{1}, \tilde{x}_{2}, \ldots, \tilde{x}_{n}\right)^{T}$ is called a fuzzy vector if $\tilde{x}_{i} \in F(\Re), \forall i=1, . ., n$.

Definition 11. Let $\tilde{A}=\left(\tilde{a}_{i j}\right)$ and $\tilde{B}=\left(\tilde{b}_{i j}\right)$ be two $m \times n$ and $n \times p$, respectively. We define $\tilde{A} \otimes \tilde{B}=\tilde{C}=\left(\tilde{c}_{i j}\right)$ which is the $m \times p$ matrix, where

$$
\tilde{c}_{i j}=\sum_{k=1, \ldots, n}^{\oplus} \tilde{a}_{i k} \otimes \tilde{b}_{k j}
$$

In this paper, if $\tilde{A}$ is the fuzzy matrix written in the form $(m, a, \beta)$, then $\tilde{A}^{\prime}$ is the symbol in the form $(a, b, c)$.

Example 2. Let $\tilde{A}, \tilde{B}$ be two fuzzy matrices in the form $(m, a, \beta)$, where

$$
\begin{aligned}
& \tilde{A}=\left(\begin{array}{ccc}
(-3,5,7) & (2,0,1) & (2,3,1) \\
(-5,4,4) & (5,3,1) & (5,6,1) \\
(6,6,6) & (-7,2,1) & (0,0,0) \\
(0,0,0) & (1,0,3) & (2,2,1)
\end{array}\right) \\
& \tilde{B}=\left(\begin{array}{cc}
(-3,2,1) & (-2,0,1) \\
(-5,1,4) & (3,3,1) \\
(4,5,1) & (-2,2,5)
\end{array}\right)
\end{aligned}
$$

Find

1. $\tilde{A} \otimes \tilde{B}$ (in the form $(m, a, \beta)$ ).

2. $\tilde{A}^{\prime} \otimes \tilde{B}^{\prime}$ (in the form $(a, b, c)$ ).

\section{Solution}

1. $\tilde{A} \otimes \tilde{B}=\tilde{C}$, (in the form $(m, a, \beta))$

$$
\begin{gathered}
\left(\begin{array}{ccc}
(-3,5,7) & (2,0,1) & (2,3,1) \\
(-5,4,4) & (5,3,1) & (5,6,1) \\
(6,6,6) & (-7,2,1) & (0,0,0) \\
(0,0,0) & (1,0,3) & (2,2,1)
\end{array}\right) \otimes\left(\begin{array}{cc}
(-3,2,1) & (-2,0,1) \\
(-5,1,4) & (3,3,1) \\
(4,5,1) & (-2,2,5)
\end{array}\right) \\
=\left(\begin{array}{cc}
(7,50,46) & (8,28,29) \\
(10,50,63) & (15,38,45) \\
(17,71,37) & (-33,27,33) \\
(3,30,11) & (-1,11,26)
\end{array}\right)
\end{gathered}
$$


2. $\tilde{A}^{\prime} \otimes \tilde{B}^{\prime}=\tilde{C},^{\prime}($ in the form $(a, b, c))$

$$
\begin{gathered}
\left(\begin{array}{ccc}
(-8,-3,4) & (2,2,3) & (-1,2,3) \\
(-9,-5,-1) & (2,5,6) & (-1,5,6) \\
(0,6,12) & (-9,-7,-6) & (0,0,0) \\
(0,0,0) & (1,1,4) & (0,2,3)
\end{array}\right) \otimes\left(\begin{array}{cc}
(-5,-3,-2) & (-2,-2,-1) \\
(-6,-5,-1) & (0,3,4) \\
(-1,4,5) & (-4,-2,3)
\end{array}\right) \\
=\left(\begin{array}{cc}
(-43,7,53) & (-20,8,37) \\
(-40,10,73) & (-23,15,60) \\
(-54,17,54) & (-60,-33,0) \\
(-27,3,14) & (-12,-1,25)
\end{array}\right)
\end{gathered}
$$

Remark 1. For determining the distance between two fuzzy vectors, the metric proposed in [37] for triangular fuzzy number is used.

If $\tilde{a}=(a, \alpha, \beta)$ and $\tilde{b}=(b, \gamma, \eta)$ are two triangular fuzzy numbers, then Ming et al. in [37] introduced the distance function

$$
D_{2}^{2}(\tilde{a}, \tilde{b})=\left(\frac{1}{2}\right)\left(4(a-b)^{2}+(\alpha-\gamma)^{2}+(\beta-\eta)^{2}\right)+(a-b)(\gamma+\eta-\alpha-\beta) .
$$

For two LR fuzzy vectors $\tilde{X}=\left(\tilde{x}_{1}, \tilde{x}_{2}, \ldots, \tilde{x}_{n}\right), \tilde{Y}=\left(\tilde{x}_{1}, \tilde{x}_{2}, \ldots, \tilde{x}_{n}\right)$ defined as

$$
D_{n}^{2}(\tilde{X}, \tilde{Y})=\sum_{i=1}^{n} D_{n}^{2}(\tilde{a}, \tilde{b}) .
$$

\section{Fully fuzzy linear system}

A brief summary on solving of fully fuzzy linear system using LP technique is provided in this section.

Definition 12. (Fully fuzzy linear system) Consider the $n \times n$ linear system,

$$
\left\{\begin{array}{c}
\tilde{a}_{11} \tilde{x}_{1}+\tilde{a}_{12} \tilde{x}_{2}+\ldots \ldots+\tilde{a}_{1 n} \tilde{x}=\tilde{b}_{1} \\
\tilde{a}_{21} \tilde{x}+a_{22} \tilde{x}_{2}+\ldots \ldots .+\tilde{a}_{2 n} \tilde{x}_{n}=\tilde{b}_{2} \\
\vdots \\
\vdots \\
\tilde{a}_{n 1} \tilde{x}_{1}+\tilde{a}_{n 2} \tilde{x}_{2}+\ldots \ldots .+\tilde{a}_{n n} \tilde{x}_{n}=\tilde{x}_{n}
\end{array} .\right.
$$

where $\forall \tilde{a}_{i j}, \tilde{b}_{j} \in F(\Re)$. This system is called a fully fuzzy linear system (FFLS).

The matrix $\tilde{A}=\left(\tilde{a}_{i j}\right)_{i, j=1}^{n}$ and the vector $\tilde{B}=\left(\tilde{b}_{j}\right)_{j=1}^{n}$ may be represented as

$$
\tilde{A} \otimes \tilde{X}=\tilde{B}
$$

The vector $\tilde{X}=\left(\tilde{x}_{j}\right)_{j=1}^{n}$ is called exact fuzzy solution if $\forall \tilde{x}_{j} \in F(\Re), j=1,2, \ldots, n$. Otherwise, it is called non-fuzzy solution.

Linear programming (LP) of solving FFLS, pioneered by Dehghan et al. in [12], is used to solve non-square FFLS for positive coefficients and parameters where the FFLS is not square, or where the size system is $n \geq 5$.

In the prior studies, LP technique was employed heavily to solve unrestricted FFLS, where at least one coefficient or parameter is near the zero TFN, because in LP we can add constraints for the system when the proposed method got certain limitations $[12,32,33,38]$.

Kumar et al. in [33] utilized LP to solve FFLS where the coefficients or parameters are unrestricted in positivity, while Babbar et al. in [32] introduced some methods in order to obtain 
the solution for some states of FFLS, and they used LP to solve the unrestricted parameters, where the coefficients are positive; the LP technique is similar to Kumar et al.'s in [33].

In both studies, the authors use LP to interpret the nature of FFLS solutions; the possible solution for FFLS may be no solution, unique, or many infinite solutions. To invalidate that, we take some of the numerical example in these studies and resolve it by original associated linear system for FFLS, and then we find further exact solutions.

Remark 2. The natures of the solutions of the FFLS depend on the nature of the solutions of the LP, i.e., the possible way of solution for FFLS may be no solution or unique, or infinitely many solutions $[32,33]$.

Because the methods depend on LP, with added further constraints of subject in order to get optimal solution, this leads to more limitations in the final solution. These algorithms find an optimal solution that is an exact solution where the objective function goes to zero. In the following examples, we will show that optimal solution is not necessarily a unique solution like in the linear system, because some or infinite solutions may be omitted and they do not appear in these algorithms.

Moreover, to the best of the researchers' knowledge, all examples which consist nearzero TFN (unrestricted FFLS) in the literature are limited by size to $n=2$, but in this paper, we provide two examples of size $n=3$.

\section{Numerical examples}

In order to show the insufficiency in LP in solving FFLS, some examples in this section are discussed. We used the LP technique in Babbar et al. in [32] to obtain the algorithm for solving a $1 \times 1$ FFLS. We find only one solution obtained by the LP technique, while the FFLS has many infinite solutions which is contrary to Remark 2.

Assuming $1 \times 1$ FFLS,

$$
\left(m_{1,1}^{a}, \alpha_{1,1}^{a}, \beta_{1,1}^{a}\right) \otimes\left(m_{1}^{x}, \alpha_{1}^{x}, \beta_{1}^{x}\right)=\left(m_{1}^{b}, \alpha_{1}^{b}, \beta_{1}^{b}\right)
$$

where $\left(m_{1,1}^{a}, \alpha_{1}^{a}, 1, \beta_{1,1}^{a}\right)$ is a non-negative triangular fuzzy number and $\tilde{x}_{1}=$ $\left(m_{1}^{x}, \alpha_{1}^{x}, \beta_{1}^{x}\right)$ is an arbitrary triangular fuzzy number.

According to algorithms in [32], to obtain the solution for the following FFLS, we have to minimize the $\bar{Z}$,

$$
\bar{Z}=Z_{1}+Z_{1}^{\prime}+Z_{1}
$$

and subject to

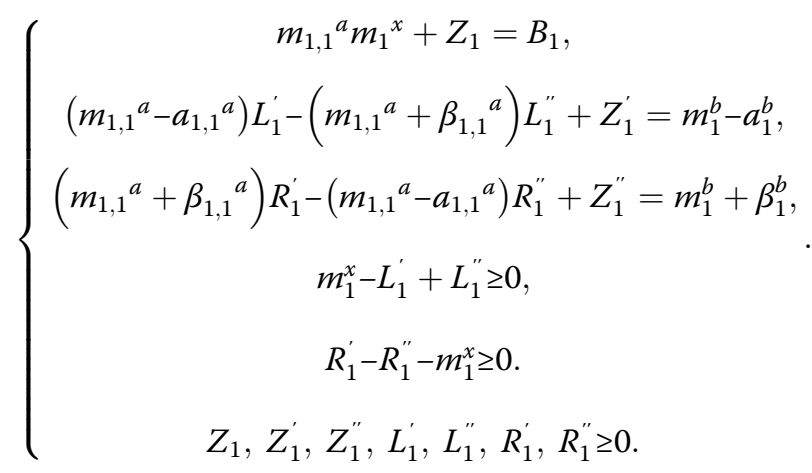


Then, the solution is obtained as follows:

$$
\tilde{x}_{1}=\left(m_{1}^{x}, \alpha_{1}^{x}, \beta_{1}^{x}\right)=\left(m_{1}^{x}, m_{1}{ }^{x}-L_{1}^{\prime}+L_{1}^{\prime \prime}, R_{1}^{\prime}-R_{1}^{\prime \prime}-m_{1}{ }^{x}\right) .
$$

The system meets the necessary and sufficient conditions for possibility of a feasible fuzzy solution, when it satisfies

$$
L_{1}^{\prime} L_{1}^{\prime \prime}=R_{1}^{\prime} R_{1}^{\prime \prime}=\bar{Z}=0 .
$$

In order to show the limitations in LP method, the algorithm is applied in the following numerical examples.

Example 3. Consider the following $1 \times 1$ FFLS, (written in the form $(m, a, \beta)$ ),

$$
(5,5,12) \otimes\left(m_{1}^{x}, \alpha_{1}^{x}, \beta_{1}^{x}\right)=(10,10,25),
$$

where $\tilde{x}_{1}=\left(m_{1}^{x}, \alpha_{1}^{x}, \beta_{1}^{x}\right)$ is an arbitrary triangular fuzzy number.

\section{Solution}

Clearly, the $(5,5,12)$ is a non-negative LR fuzzy number. According to [32], we can apply their method to solve the system with no restrictions on the solution.

Now, let

$$
\begin{aligned}
& m_{1,1}{ }^{a}=5, \alpha_{1,1}{ }^{a}=5, \quad \beta_{1,1}{ }^{a}=12, \\
& m_{1}{ }^{b}=10, \quad \alpha_{1}{ }^{b}=10, \beta_{1}{ }^{b}=25 .
\end{aligned}
$$

Then, minimization of problem in (14) is

$$
5 m_{1}^{x}+Z_{1}=10 .
$$

The subject in (15) is

$$
\left\{\begin{array}{l}
-17 L_{1}^{\prime \prime}+Z_{1}^{\prime}=0, \\
17 R_{1}^{\prime}+Z_{1}^{\prime \prime}=35, \\
m_{1}^{x}-L_{1}^{\prime}+L_{1}^{\prime \prime} \geq 0, \\
R_{1}^{\prime}-R_{1}^{\prime \prime}-m_{1}^{x} \geq 0, \\
Z_{1}, Z_{1}^{\prime}, Z_{1}^{\prime \prime}, L_{1}^{\prime}, L_{1}^{\prime \prime}, R_{1}^{\prime}, R_{1}^{\prime \prime} \geq 0 .
\end{array}\right.
$$

On solving the above LP, the following solution is obtained:

$$
m_{1}^{x}=2, L_{1}^{\prime}=2, L_{1}^{\prime \prime}=0, R_{1}^{\prime}=\frac{35}{17}, R_{1}^{\prime \prime}=0,
$$

Also, the objective function in (14) is zero since

$$
Z_{1}=Z_{1}^{\prime}=Z_{1}^{\prime \prime}=0 \text {, then } \bar{Z}=0 .
$$

Similarly, in (17), the right-hand side is zero,

$$
L_{1}^{\prime} L_{1}^{\prime \prime}=R_{1}^{\prime} R_{1}^{\prime \prime}=\bar{Z}=0 .
$$

Hence, the system has feasible fuzzy solution, using (16) and (20)

$$
\left(m_{1}^{x}, \alpha_{1}^{x}, \beta_{1}^{x}\right)=\left(2,2-0+0, \frac{35}{17}-0-2\right) \text {. }
$$


Then the solution of FFLS in (18) becomes

$$
\tilde{X}_{k}=\tilde{x}_{1}=\left(m_{1}^{x}, \alpha_{1}^{x}, \beta_{1}^{x}\right)=\left(2,0, \frac{1}{17}\right) .
$$

However, the following general form solution confirms that the system has many infinite solutions which are not detected using previous algorithm by LP,

$$
\left(m_{1}^{x}, \alpha_{1}^{x}, \beta_{1}^{x}\right)=\left(2, \omega, \frac{1}{17}\right),
$$

where $\omega \in[0,2]$.

Thus, the solution $\tilde{X}_{k}$ obtained by the algorithm in [32] using LP is a particular solution only where $\omega=0$, in (24).

The following are some solutions using (24),

$$
\begin{aligned}
& \tilde{x}_{1}=\left(m_{1}{ }^{x}, \alpha_{1}{ }^{x}, \beta_{1}{ }^{x}\right)=\left(2,2, \frac{1}{17}\right), \\
& \tilde{x}_{2}=\left(m_{1}{ }^{x}, \alpha_{1}{ }^{x}, \beta_{1}{ }^{x}\right)=\left(2,1, \frac{1}{17}\right), \\
& \tilde{x}_{3}=\left(m_{1}{ }^{x}, \alpha_{1}{ }^{x}, \beta_{1}{ }^{x}\right)=\left(2, \frac{3}{2}, \frac{1}{17}\right), \\
& \tilde{x}_{4}=\left(m_{1}{ }^{x}, \alpha_{1}{ }^{x}, \beta_{1}{ }^{x}\right)=\left(2, \frac{1}{2}, \frac{1}{17}\right) .
\end{aligned}
$$

It is clear that the system has infinitely many feasible fuzzy solutions, which is contrary to the nature of possible way solution for the linear system, where every linear system in order $1 \times 1$ must have a unique solution.

On the other hand, the following Example 4 gives us a different result from Example 3 , because it is non-square FFLS, where the number of equations is more than the parameters, and the system has a unique solution.

Example 4. Consider the following $2 \times 1$ FFLS (written in the form $(m, \alpha, \beta))$ :

$$
\left\{\begin{array}{l}
(3,1,1) \otimes\left(m_{1}^{x}, \alpha_{1}{ }^{x}, \beta_{1}^{x}\right)=(0,24,12), \\
(2,1,0) \otimes\left(m_{1}^{x}, \alpha_{1}^{x}, \beta_{1}{ }^{x}\right)=(0,12,6),
\end{array}\right.
$$

where $\tilde{X}=\tilde{x}_{1}=\left(m_{1}^{x}, \alpha_{1}^{x}, \beta_{1}^{x}\right)$, is an arbitrary TFN.

Solution

The FFLS may be written in matrix form $\tilde{A} \otimes \tilde{X}=\tilde{B}$,

$$
\left(\begin{array}{l}
(3,1,1) \\
(2,1,0)
\end{array}\right) \otimes\left(\tilde{x}_{1}\right)=\left(\begin{array}{c}
(0,24,12) \\
(0,12,6)
\end{array}\right)
$$

The system has a unique solution,

$$
\tilde{X}=\left(m_{1}{ }^{x}, \alpha_{1}{ }^{x}, \beta_{1}{ }^{x}\right)=(0,6,3) .
$$


Verification for the solution:

$$
\left\{\begin{array}{l}
(3,1,1) \otimes(0,6,3)=(0,24,12), \\
(2,1,0) \otimes(0,6,3)=(0,12,6) .
\end{array}\right.
$$

The following non-square FFLS has many infinite solutions where the number of equations is less than the number of parameters, the result contrary to the result in Example 4.

Example 5. ([33]) Consider the following $2 \times 3$ FFLS (written in the form $(a, b, c)$ ):

$$
\left\{\begin{array}{c}
(1,2,3) \otimes\left(a_{1}^{x}, b_{1}{ }^{x}, c_{1}^{x}\right) \oplus(-2,-1,-1) \otimes\left(a_{2}{ }^{x}, b_{2}{ }^{x}, c_{2}{ }^{x}\right) \\
\oplus(2,3,4) \otimes\left(a_{3}^{x}, b_{3}{ }^{x}, c_{3}{ }^{x}\right)=(-7,0,8), \\
(-3,-2,-1) \otimes\left(a_{1}^{x}, b_{1}^{x}, c_{1}^{x}\right) \oplus(2,4,5) \otimes\left(a_{2}{ }^{x}, b_{2}{ }^{x}, c_{2}{ }^{x}\right) \\
\oplus(3,4,5) \otimes\left(a_{3}{ }^{x}, b_{3}{ }^{x}, c_{3}{ }^{x}\right)=(-26,-10,-4),
\end{array}\right.
$$

where $\tilde{x}_{i}{ }^{\prime}=\left(a_{i}^{x}, b_{i}{ }^{x}, c_{i}{ }^{x}\right), i=1,2,3$, are arbitraries triangular fuzzy numbers.

Solution

The system may be written in matrix form $\tilde{A}^{\prime} \otimes \tilde{X}^{\prime}=\tilde{B}^{\prime}$,

$$
\left(\begin{array}{ccc}
(1,2,3) & (-2,-1,-1) & (2,3,4) \\
(-3,-2,-1) & (2,4,5) & (3,4,5)
\end{array}\right) \otimes\left(\begin{array}{c}
\tilde{x}_{1}^{\prime} \\
\tilde{x}_{2}^{\prime} \\
\tilde{x}_{3}^{\prime}
\end{array}\right)=\left(\begin{array}{c}
(-7,0,8) \\
(-26,-10,-4)
\end{array}\right)
$$

where

$$
\tilde{X}_{k}^{\prime}=\left(\begin{array}{c}
\tilde{x}_{1}^{\prime} \\
\tilde{x}_{2}^{\prime} \\
\tilde{x}_{3}^{\prime}
\end{array}\right)=\left(\begin{array}{c}
\left(a_{1}^{x}, b_{1}^{x}, c_{1}^{x}\right) \\
\left(a_{2}^{x}, b_{2}^{x}, c_{2}^{x}\right) \\
\left(a_{3}^{x}, b_{3}^{x}, c_{3}^{x}\right)
\end{array}\right)
$$

According to Kumar et al. in [33], the system has one solution, since LP technique produces one optimal solution; for that the fuzzy vector, $\tilde{X}_{k}{ }_{k}$ is provided as a particular solution:

$$
\tilde{X}_{k}^{\prime}=\left(\begin{array}{c}
\left(a_{1}^{x}, b_{1}^{x}, c_{1}{ }^{x}\right) \\
\left(a_{2}{ }^{x}, b_{2}{ }^{x}, c_{2}{ }^{x}\right) \\
\left(a_{3}{ }^{x}, b_{3}{ }^{x}, c_{3}{ }^{x}\right)
\end{array}\right)=\left(\begin{array}{c}
(1,1,2) \\
(-2,-1,0) \\
(-2,-1,-1)
\end{array}\right) \text {. }
$$

By solving the associated linear system, we provide the general form solution, which cannot be obtained by LP, and then some particular solutions are produced: 


$$
\tilde{X}_{G^{\prime}}=\left(\begin{array}{c}
\left(a_{1}{ }^{x}, b_{1}{ }^{x}, c_{1}{ }^{x}\right) \\
\left(a_{2}{ }^{x}, b_{2}{ }^{x}, c_{2}{ }^{x}\right) \\
\left(a_{3}{ }^{x}, b_{3}{ }^{x}, c_{3}{ }^{x}\right)
\end{array}\right)=\left(\begin{array}{c}
\left(1, \frac{1}{7}(15+8 \delta), 2\right) \\
(-2, \delta, 0) \\
\left(-2, \frac{1}{3}\left(\delta-\frac{2}{7}(15+8 \delta)\right),-1\right)
\end{array}\right),
$$

where $\delta \in\left[-1,-\frac{1}{8}\right]$

Thus, the solution $\tilde{X}_{k}{ }^{\prime}$ obtained by the algorithm in Kumar et al. [33] using LP is a particular solution only where $\delta=-1$, in (27).

Some particular solutions:

- Let $\delta=-\frac{1}{8}$, then

$$
\tilde{X}_{1}^{\prime}=\left(\begin{array}{c}
\left(a_{1}{ }^{x}, b_{1}{ }^{x}, c_{1}{ }^{x}\right) \\
\left(a_{2}{ }^{x}, b_{2}{ }^{x}, c_{2}{ }^{x}\right) \\
\left(a_{3}{ }^{x}, b_{3}{ }^{x}, c_{3}{ }^{x}\right)
\end{array}\right)=\left(\begin{array}{c}
(1,2,2) \\
\left(-2,-\frac{1}{8}, 0\right) \\
\left(-2,-\frac{11}{8},-1\right)
\end{array}\right) .
$$

Verification for the solution:

$$
\left\{\begin{array}{c}
(1,2,3) \otimes(1,2,2) \oplus(-2,-1,-1) \otimes\left(-2,-\frac{1}{8}, 0\right) \oplus(2,3,4) \otimes\left(-2,-\frac{11}{8},-1\right) \\
=(1,4,6) \oplus\left(0, \frac{1}{8}, 4\right) \oplus\left(-8,-\frac{33}{8},-2\right)=(-7,0,8), \\
(-3,-2,-1) \otimes(1,2,2) \oplus(2,4,5) \otimes\left(-2,-\frac{1}{8}, 0\right) \oplus(3,4,5) \otimes\left(-2,-\frac{11}{8},-1\right) \\
=(-6,-4,-1) \oplus\left(-10,-\frac{11}{2},-3\right)=(-26,-10,-4)
\end{array}\right.
$$

- Let $\delta=-\frac{1}{2}$, then

$$
\tilde{X}_{2}^{\prime}=\left(\begin{array}{c}
\left(a_{1}{ }^{x}, b_{1}{ }^{x}, c_{1}{ }^{x}\right) \\
\left(a_{2}{ }^{x}, b_{2}{ }^{x}, c_{2}{ }^{x}\right) \\
\left(a_{3}{ }^{x}, b_{3}{ }^{x}, c_{3}{ }^{x}\right)
\end{array}\right)=\left(\begin{array}{c}
\left(1, \frac{11}{7}, 2\right) \\
\left(-2,-\frac{1}{2}, 0\right) \\
\left(-2,-\frac{17}{14},-1\right)
\end{array}\right) .
$$


Verification for the solution:

$$
\left\{\begin{array}{l}
(1,2,3) \otimes\left(1, \frac{11}{7}, 2\right) \oplus(-2,-1,-1) \otimes\left(-2,-\frac{1}{2}, 0\right) \oplus(2,3,4) \otimes\left(-2,-\frac{17}{14},-1\right) \\
=\left(1, \frac{22}{7}, 6\right) \oplus\left(0, \frac{1}{2}, 4\right) \oplus\left(-8, \frac{-51}{14},-2\right) \\
=(-7,0,8),(-3,-2,-1) \otimes\left(1, \frac{11}{7}, 2\right) \oplus(2,4,5) \otimes\left(-2,-\frac{1}{2}, 0\right) \oplus(3,4,5) \otimes\left(-2,-\frac{17}{14},-1\right) \\
=\left(-6,-\frac{22}{7},-1\right) \oplus(-10,-2,0) \oplus\left(-10,-\frac{34}{7},-3\right)=(-26,-10,-4) .
\end{array}\right.
$$

- Let $\delta=-\frac{1}{3}$, then

$$
\tilde{X}_{3}{ }^{\prime}=\left(\begin{array}{c}
\left(a_{1}{ }^{x}, b_{1}{ }^{x}, c_{1}{ }^{x}\right) \\
\left(a_{2}{ }^{x}, b_{2}{ }^{x}, c_{2}{ }^{x}\right) \\
\left(a_{3}{ }^{x}, b_{3}{ }^{x}, c_{3}{ }^{x}\right)
\end{array}\right)=\left(\begin{array}{c}
\left(1, \frac{37}{21}, 2\right) \\
\left(-2,-\frac{1}{3}, 0\right) \\
\left(-2,-\frac{9}{7},-1\right)
\end{array}\right) .
$$

Verification for the solution:

$$
\left\{\begin{array}{l}
(1,2,3) \otimes\left(1, \frac{37}{21}, 2\right) \oplus(-2,-1,-1) \otimes\left(-2,-\frac{1}{3}, 0\right) \oplus(2,3,4) \otimes\left(-2,-\frac{9}{7},-1\right) \\
=\left(1, \frac{74}{21}, 6\right) \oplus\left(0, \frac{1}{3}, 4\right) \oplus\left(-8,-\frac{27}{7},-2\right) \\
=(-7,0,8),(-3,-2,-1) \otimes\left(1, \frac{37}{21}, 2\right) \oplus(2,4,5) \otimes\left(-2,-\frac{1}{3}, 0\right) \oplus(3,4,5) \otimes\left(-2,-\frac{9}{7},-1\right) \\
=\left(-6,-\frac{74}{21},-1\right) \oplus\left(-10,-\frac{4}{3}, 0\right) \oplus\left(-10,-\frac{36}{7},-3\right)=(-26,-10,-4)
\end{array}\right.
$$

then

$$
\tilde{A}^{\prime} \otimes \tilde{X}_{i}^{\prime}=\tilde{A}^{\prime} \otimes \tilde{X}_{k}{ }^{\prime}=\tilde{B}^{\prime}, i=1, \ldots, 3
$$

- Non-fuzzy solution

$$
\delta \notin\left[-1,-\frac{1}{8}\right] \text { where } \delta=-\frac{3}{2} \text {, then }
$$




$$
\tilde{X}_{e}^{\prime}=\left(\begin{array}{c}
\left(a_{1}^{x}, b_{1}{ }^{x}, c_{1}{ }^{x}\right) \\
\left(a_{2}{ }^{x}, b_{2}{ }^{x}, c_{2}{ }^{x}\right) \\
\left(a_{3}{ }^{x}, b_{3}{ }^{x}, c_{3}{ }^{x}\right)
\end{array}\right)=\left(\begin{array}{c}
\left(1, \frac{3}{7}, 2\right) \\
\left(-2,-\frac{3}{2}, 0\right) \\
\left(-2,-\frac{11}{14},-1\right)
\end{array}\right) .
$$

Verification for the solution:

$$
\left\{\begin{array}{l}
(1,2,3) \otimes\left(1, \frac{15}{7}, 2\right) \oplus(-2,-1,-1) \otimes(-2,0,0) \oplus(2,3,4) \otimes\left(-2,-\frac{10}{7},-1\right) \\
=\left(1, \frac{30}{7}, 6\right) \oplus(0,0,4) \oplus\left(-8,-\frac{30}{7},-2\right) \\
=(-7,0,8)(-3,-2,-1) \otimes\left(1, \frac{15}{7}, 2\right) \oplus(2,4,5) \otimes(-2,0,0) \oplus(3,4,5) \otimes\left(-2,-\frac{10}{7},-1\right) \\
=\left(-6,-\frac{30}{7},-1\right) \oplus(-10,0,0) \oplus\left(-10,-\frac{40}{7},-3\right)=(-26,-10,-4) .
\end{array}\right.
$$

Although $\tilde{X}_{e}{ }^{\prime}$ satisfies the system,

$$
\tilde{A}^{\prime} \otimes \tilde{X}_{e}{ }^{\prime}=\tilde{B}^{\prime} .
$$

But it is not a feasible solution, because $b_{3}^{x} / c_{3}^{x}$, then $\tilde{X}_{k}{ }^{\prime}$ is not a fuzzy vector.

Now, the examples are converted to the form in this paper $(m, \alpha, \beta)$,

$$
\begin{aligned}
\tilde{A}= & \left(\begin{array}{ll}
\left(m_{1,1}^{a}, a_{1,1}{ }^{a}, \beta_{1,1}{ }^{a}\right)\left(m_{2,1^{a}}, a_{2,1}{ }^{a}, \beta_{2,1}{ }^{a}\right)\left(m_{3,1}{ }^{a}, a_{3,1}{ }^{a}, \beta_{3,1}{ }^{a}\right) \\
\left(m_{1,2}{ }^{a}, a_{1,2}, \beta_{1,2}{ }^{a}\right)\left(m_{2,2}{ }^{a}, a_{2,2}, \beta_{2,2}{ }^{a}\right)\left(m_{3,2}{ }^{a}, a_{3,2}{ }^{a}, \beta_{3,2}{ }^{a}\right)
\end{array}\right)= \\
& \left(\begin{array}{lll}
(2,1,1) & (-1,1,0) & (3,1,1) \\
(-2,1,1) & (4,2,1) & (4,1,1)
\end{array}\right)
\end{aligned}
$$

and

$$
\tilde{B}=\left(\begin{array}{c}
\left(m_{1}^{b}, a_{1}^{b}, \beta_{1}^{b}\right) \\
\left(m_{2}^{b}, a_{2}^{b}, \beta_{2}^{b}\right)
\end{array}\right)=\left(\begin{array}{c}
(0,7,14) \\
(-10,16,14)
\end{array}\right),
$$

then the system in (26) is equivalent to the following system:

$$
\left(\begin{array}{lrr}
(2,1,1) & (-1,1,0) & (3,1,1) \\
(-2,1,1) & (4,2,1) & (4,1,1)
\end{array}\right) \otimes\left(\begin{array}{c}
\tilde{x}_{1} \\
\tilde{x}_{2} \\
\tilde{x}_{2}
\end{array}\right)=\left(\begin{array}{c}
(0,7,14) \\
(-10,16,14)
\end{array}\right),
$$

Where

$$
\tilde{X}=\left(\begin{array}{c}
\tilde{x}_{1} \\
\tilde{x}_{2} \\
\tilde{x}_{2}
\end{array}\right)=\left(\begin{array}{c}
\left(m_{1}^{x}, a_{1}^{x}, \beta_{1}{ }^{x}\right) \\
\left(m_{2}^{x}, a_{2}^{x}, \beta_{2}{ }^{x}\right) \\
\left(m_{3}^{x}, a_{3}^{x}, \beta_{3}{ }^{x}\right)
\end{array}\right) .
$$


The produced solution in [32]

$$
\tilde{X}_{k}=\left(\begin{array}{c}
\left(m_{1}^{x}, a_{1}^{x}, \beta_{1}{ }^{x}\right) \\
\left(m_{2}^{x}, a_{2}{ }^{x}, \beta_{2}{ }^{x}\right) \\
\left(m_{3}^{x}, a_{3}{ }^{x}, \beta_{3}{ }^{x}\right)
\end{array}\right)=\left(\begin{array}{c}
(1,0,1) \\
(-1,1,1) \\
(-1,1,0)
\end{array}\right) .
$$

The general form solution is

$$
\begin{aligned}
& \tilde{X}_{G}=\left(\begin{array}{c}
\left(m_{1}^{x}, a_{1}^{x}, \beta_{1}^{x}\right) \\
\left(m_{2}^{x}, a_{2}^{x}, \beta_{2}^{x}\right) \\
\left(m_{3}^{x}, a_{3}^{x}, \beta_{3}^{x}\right)
\end{array}\right)= \\
& \left(\begin{array}{c}
\left(\frac{1}{7}(15+8 \delta)\right),-1+\frac{1}{7}(15+8 \delta), 2+\frac{1}{7}(-15-8 \delta) \\
(\delta, 2+\delta,-\delta) \\
\left(\frac{1}{3}\left(\delta-\frac{2}{7}(15+8 \delta)\right), 2+\frac{1}{3}\left(\delta-\frac{2}{7}(15+8 \delta)\right),-1+\frac{1}{3}\left(-\delta+\frac{2}{7}(15+8 \delta)\right)\right)
\end{array}\right)
\end{aligned}
$$

The particular solutions are

$$
\begin{aligned}
& \tilde{X}_{1}=\left(\begin{array}{l}
\left(m_{1}^{x}, a_{1}{ }^{x}, \beta_{1}{ }^{x}\right) \\
\left(m_{2}{ }^{x}, a_{2}{ }^{x}, \beta_{2}{ }^{x}\right) \\
\left(m_{3}{ }^{x}, a_{3}{ }^{x}, \beta_{3}{ }^{x}\right)
\end{array}\right)=\left(\begin{array}{c}
(2,1,0) \\
\left(-\frac{1}{8}, \frac{15}{8}, \frac{1}{8}\right) \\
\left(-\frac{11}{8}, \frac{5}{8}, \frac{3}{8}\right)
\end{array}\right), \\
& \tilde{X}_{2}=\left(\begin{array}{c}
\left(m_{1}^{x}, a_{1}^{x}, \beta_{1}{ }^{x}\right) \\
\left(m_{2}{ }^{x}, a_{2}{ }^{x}, \beta_{2}{ }^{x}\right) \\
\left(m_{3}{ }^{x}, a_{3}{ }^{x}, \beta_{3}{ }^{x}\right)
\end{array}\right)=\left(\begin{array}{c}
\left(\frac{11}{7}, \frac{4}{7}, \frac{3}{7}\right) \\
\left(-\frac{1}{2}, \frac{3}{2}, \frac{1}{2}\right) \\
\left(-\frac{17}{14}, \frac{11}{14}, \frac{3}{14}\right)
\end{array}\right), \\
& \tilde{X}_{3}=\left(\begin{array}{l}
\left(m_{1}^{x}, a_{1}{ }^{x}, \beta_{1}{ }^{x}\right) \\
\left(m_{2}{ }^{x}, a_{2}{ }^{x}, \beta_{2}{ }^{x}\right) \\
\left(m_{3}{ }^{x}, a_{3}{ }^{x}, \beta_{3}{ }^{x}\right)
\end{array}\right)=\left(\begin{array}{c}
\left(\frac{37}{21}, \frac{16}{21}, \frac{5}{21}\right) \\
\left(-\frac{1}{3}, \frac{5}{3}, \frac{1}{3}\right) \\
\left(-\frac{9}{7}, \frac{5}{7}, \frac{2}{7}\right)
\end{array}\right) .
\end{aligned}
$$


The non-fuzzy solution is

$$
\tilde{X}_{e}=\left(\begin{array}{c}
\left(m_{1}{ }^{x}, a_{1}{ }^{x}, \beta_{1}{ }^{x}\right) \\
\left(m_{2}{ }^{x}, a_{2}{ }^{x}, \beta_{2}{ }^{x}\right) \\
\left(m_{3}{ }^{x}, a_{3}{ }^{x}, \beta_{3}{ }^{x}\right)
\end{array}\right)=\left(\begin{array}{c}
\left(\frac{3}{7},-\frac{4}{7}, \frac{11}{7}\right) \\
\left(-\frac{3}{2}, \frac{1}{2}, \frac{3}{2}\right) \\
\left(-\frac{11}{14}, \frac{17}{14},-\frac{3}{14}\right)
\end{array}\right) .
$$

Remark 3. The nature of uniqueness solution or infinity is completely different with the nature of the linear system or linear programing because the number of feasible solutions does not depend on the number of equations compared with number of parameters.

The case of no solution is considered by the following example even when the system has one equation with one parameter, contrary to Example 3 and Example 7.

Example 6. Consider the following $1 \times 1$ FFLS (written in the form $(m, \alpha, \beta)$ ).

$$
(3,1,1) \otimes\left(m_{1}^{x}, a_{1}{ }^{x}, \beta_{1}{ }^{x}\right)=(-36,4,18) .
$$

where $\tilde{x}_{1}=\left(m_{1}^{x}, a_{1}{ }^{x}, \beta_{1}{ }^{x}\right)$ is an arbitrary TFN.

\section{Solution}

The exact solution of the system is a non-fuzzy solution because the right spread $\alpha_{1,1}^{x}$ is non-positive.

$$
\tilde{x}_{1}=\left(m_{1}^{x}, a_{1}^{x}, \beta_{1}^{x}\right)=(-12,-2,3) .
$$

The solution $\tilde{x}_{1}$ satisfies the system, but it is not TFN, so it is not considered as a feasible solution

Remark 4. The existence of feasible solution for FFLS is determined by condition of TFN regardless of the existence of the associated system for FFLS.

In light of the previous results, we can declare that the possible ways of solution (unique solutions, infinite solutions, no solution) in FFLS are not determined by the number of equations compared with the number of parameters, or if the FFLS is square or non-square, all possible ways may happen regardless of the number of equations and parameters.

The following examples of FFLS in size $n=1$ which is similar with the essential concept of linear system, where the number of equation is equal to the number of parameters, and they have a unique feasible solution.

Example 7. Consider the following $1 \times 1$ FFLS, written in the form $(m, \alpha, \beta)$,

$$
(5,0,12) \otimes\left(m_{1,1}^{x}, a_{1,1^{x}}, \beta_{1,1}^{x}\right)=(10,9,25),
$$

where $\tilde{x}=\left(m_{1}^{x}, a_{1}^{x}, \beta_{1}^{x}\right)$ is an arbitrary triangular fuzzy number.

\section{Solution}

The FFLS has only one solution,

$$
\tilde{x}=\left(m_{1}^{x}, a_{1}^{x}, \beta_{1}^{x}\right)=\left(2, \frac{9}{5}, \frac{1}{17}\right) .
$$

The following example is an FFLS and has also a unique solution, similar to Example 7 , where the size of the coefficient matrix is $n=3$. 
Example 8. Consider the following $3 \times 3$ FFLS (written in the form $(m, \alpha, \beta)$ ).

$$
\left\{\begin{array}{c}
(8,2,1) \otimes\left(m_{1}^{x}, a_{1}{ }^{x}, \beta_{1}{ }^{x}\right) \oplus(2,1,1) \otimes\left(m_{2}{ }^{x}, a_{2}{ }^{x}, \beta_{2}{ }^{x}\right) \\
\oplus(7,2,2) \otimes\left(m_{3}{ }^{x}, a_{3}{ }^{x}, \beta_{3}{ }^{x}\right)=(0,55,69), \\
(2,1,0) \otimes\left(m_{1}^{x}, a_{1}{ }^{x}, \beta_{1}{ }^{x}\right) \oplus(4,1,1) \otimes\left(m_{2}{ }^{x}, a_{2}{ }^{x}, \beta_{2}{ }^{x}\right) \\
\oplus(2,1,1) \otimes\left(m_{3}{ }^{x}, a_{3}{ }^{x} \beta_{3}{ }^{x}\right)=(4,51,36), \\
(5,1,2) \otimes\left(m_{1}{ }^{x}, a_{1}{ }^{x}, \beta_{1}{ }^{x}\right) \oplus(9,2,3) \otimes\left(m_{2}{ }^{x}, a_{2}{ }^{x}, \beta_{2}{ }^{x}\right) \\
\oplus(0,0,0) \otimes\left(m_{3}{ }^{x}, a_{3}{ }^{x}, \beta_{3}{ }^{x}\right)=(-1,123,45),
\end{array}\right.
$$

Where $\tilde{x}_{i}=\left(m_{i}^{x}, a_{i}{ }^{x}, \beta_{i}{ }^{x}\right), i=1,2,3$ are arbitrary triangular fuzzy numbers.

\section{Solution}

The system may be written in matrix form $\tilde{A} \otimes \tilde{X}=\tilde{B}$,

$$
\begin{aligned}
& \left(\begin{array}{lll}
(8,2,1) & (2,1,1) & (7,2,2) \\
(2,1,0) & (4,1,1) & (2,1,1) \\
(5,1,2) & (9,2,3) & (0,0,0)
\end{array}\right) \otimes\left(\begin{array}{l}
\left(m_{1}^{x}, a_{1}^{x}, \beta_{1}^{x}\right) \\
\left(m_{2}^{x}, a_{2}{ }^{x}, \beta_{2}{ }^{x}\right) \\
\left(m_{3}^{x}, a_{3}{ }^{x}, \beta_{3}{ }^{x}\right)
\end{array}\right) \\
& =\left(\begin{array}{c}
(0,55,69) \\
(4,51,36) \\
(-1,123,45)
\end{array}\right)
\end{aligned}
$$

Where

$$
\tilde{X}=\left(\begin{array}{c}
\tilde{x}_{1} \\
\tilde{x}_{2} \\
\tilde{x}_{3}
\end{array}\right)=\left(\begin{array}{c}
\left(m_{1}{ }^{x}, a_{1}{ }^{x}, \beta_{1}{ }^{x}\right) \\
\left(m_{2}{ }^{x}, a_{2}{ }^{x}, \beta_{2}{ }^{x}\right) \\
\left(m_{3}{ }^{x}, a_{3}{ }^{x}, \beta_{3}{ }^{x}\right)
\end{array}\right) \text {. }
$$

The system has a unique feasible solution:

$$
\tilde{X}=\left(\begin{array}{c}
\tilde{x}_{1} \\
\tilde{x}_{2} \\
\tilde{x}_{3}
\end{array}\right)=\left(\begin{array}{c}
\left(m_{1}{ }^{x}, a_{1}{ }^{x}, \beta_{1}{ }^{x}\right) \\
\left(m_{2}{ }^{x}, a_{2}{ }^{x}, \beta_{2}{ }^{x}\right) \\
\left(m_{3}{ }^{x}, a_{3}{ }^{x}, \beta_{3}{ }^{x}\right)
\end{array}\right)=\left(\begin{array}{c}
(-2,2,1) \\
(1,9,3) \\
(2,15)
\end{array}\right) .
$$


Verification for the solution:

$$
\left(\begin{array}{c}
(8,2,1) \otimes(-2,2,1) \oplus(2,1,1) \otimes(1,9,3) \oplus(7,2,2) \otimes(2,1,5)= \\
(-16,20,10) \oplus(2,26,10) \oplus(14,9.49)=(0,55,69) \\
(2,1,0) \otimes(-2,2,1) \oplus(4,1,1) \otimes(1,9,3) \oplus(2,1,1) \otimes(2,1,5)= \\
(-4,4,3) \oplus(4,44,16) \oplus(4,3,17)=(4,51,36), \\
(5,1,2) \otimes(-2,2,1) \oplus(9,2,3) \otimes(1,9,3) \oplus(0,0,0) \otimes(2,1,5)= \\
(-10,18,6) \oplus(9,105,39) \oplus(0,0,0)=(-1,123,45) .
\end{array}\right)
$$

In the rest of this paper, we will show new facts of the nature of the FFLS solution, which is the unique case that may be assigned two different feasible solutions on the contrary case of uniqueness in the linear system. For that, the LP does not interpret the uniqueness of the FFLS case.

To support that, we take an example from Kumar et al. in [33] and obtain further different feasible solutions, while in the proposed method, only one solution is assigned. The triangular fuzzy number in [33] is formed in $(a, b, c)$, so Example 9 and Example 10 are rewritten in both forms.

Example 9. ([33]) Consider the following $2 \times 2$ FFLS (written in form $(a, b, c)$ )

$$
\left\{\begin{array}{c}
(-2,3,4) \otimes\left(a_{1}{ }^{x}, b_{1}{ }^{x}, c_{1}{ }^{x}\right) \oplus(-2,2,3) \otimes\left(a_{2}{ }^{x}, b_{2}{ }^{x}, c_{2}{ }^{x}\right)=(-13,8,14), \\
(1,2,2) \otimes\left(a_{1}{ }^{x}, b_{1}{ }^{x}, c_{1}^{x}\right) \oplus(4,4,5) \otimes\left(a_{2}{ }^{x}, b_{2}{ }^{x}, c_{2}{ }^{x}\right)=(-14,8,14) .
\end{array}\right.
$$

\section{Solution}

The system may written in matrix form $\tilde{A}^{\prime} \otimes \tilde{X}^{\prime}=\tilde{B}^{\prime}$,

$$
\left(\begin{array}{cc}
(-2,3,4) & (-2,2,3) \\
(1,2,2) & (4,4,5)
\end{array}\right) \otimes\left(\begin{array}{c}
\tilde{x}_{1} \\
\tilde{x}_{2}
\end{array}\right)=\left(\begin{array}{c}
(-13,8,14) \\
(-14,8,14)
\end{array}\right)
$$

where

$$
\tilde{X}=\left(\begin{array}{c}
\tilde{x}_{1}{ }^{\prime} \\
\tilde{x}_{2}^{\prime}
\end{array}\right)=\left(\begin{array}{c}
\left(a_{1}^{x}, b_{1}^{x}, c_{1}^{x}\right) \\
\left(a_{2}^{x}, b_{2}^{x}, c_{2}^{x}\right)
\end{array}\right) .
$$

$\tilde{X}_{i}{ }^{\prime}=\left(a_{i}{ }^{x}, b_{i}{ }^{x}, c_{i}{ }^{x}\right), i=1,2$ are arbitrary triangular fuzzy numbers.

The solution in Kumar et al. [33] is unique and is as follows:

$$
\tilde{X}_{k}^{\prime}=\left(\begin{array}{c}
\tilde{x}_{1}^{\prime} \\
\tilde{x}_{2}^{\prime}
\end{array}\right)=\left(\begin{array}{c}
\left(a_{1}^{x}, b_{1}^{x}, c_{1}^{x}\right) \\
\left(a_{2}^{x}, b_{2}^{x}, c_{2}^{x}\right)
\end{array}\right)=\left(\begin{array}{c}
(1,2,2) \\
(-3,1,2)
\end{array}\right) .
$$

While the solution, $\tilde{X}_{g}$ 'represents further solution which cannot be determined through the LP method.

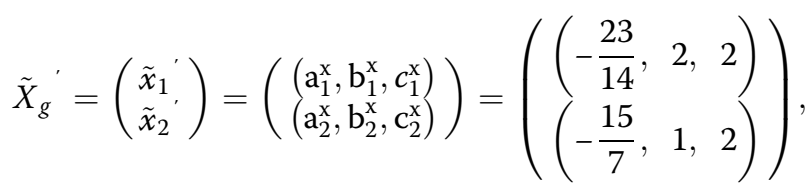


Verification for the solution:

$$
\left\{\begin{aligned}
(-2,3,4) & \otimes \\
= & \left(-\frac{23}{14}, 2,2\right) \oplus(-2,2,3) \otimes\left(-\frac{15}{7}, 1,2\right) \\
(1,2,2) & \otimes\left(-\frac{23}{14}, 2,2\right) \oplus(4,4,5) \otimes\left(-\frac{15}{7}, 1,2\right) \\
= & \left(-\frac{23}{7}, 4,4\right) \oplus\left(-\frac{75}{7}, 2,6\right)=(-13,8,14),
\end{aligned}\right.
$$

Because we follow the $(m, \alpha, \beta)$ form for TFN in this paper, the example is converted to this form.

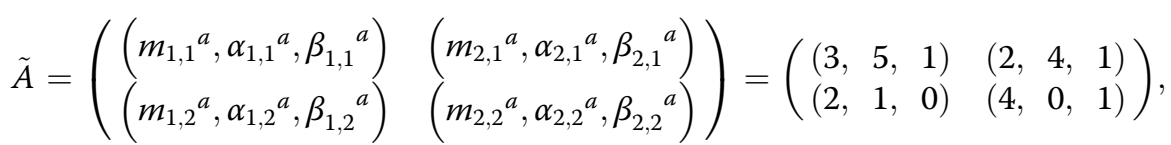

where

$$
\begin{aligned}
\tilde{X} & =\left(\begin{array}{c}
\tilde{x}_{1} \\
\tilde{x}_{2}
\end{array}\right)=\left(\begin{array}{c}
\left(m_{1}^{x}, \alpha_{1}^{x}, \beta_{1}^{x}\right) \\
\left(m_{2}^{x}, \alpha_{2}^{x}, \beta_{2}^{x}\right)
\end{array}\right), \\
\tilde{x}_{i} & =\left(m_{i}^{x}, \alpha_{i}^{x}, \beta_{i}^{x}\right), i=1,2 \text { are arbitrary triangular fuzzy numbers, } \\
\tilde{B} & =\left(\begin{array}{c}
\left(m_{1}^{b}, \alpha_{1}^{b}, \beta_{1}{ }^{b}\right) \\
\left(m_{2}^{b}, \alpha_{2}^{b}, \beta_{2}^{b}\right)
\end{array}\right)=\left(\begin{array}{c}
(8,21,6) \\
(8,22,6)
\end{array}\right)
\end{aligned}
$$

then the FFLS in (34) is equivalent to the following matrix form $\tilde{A} \otimes \tilde{X}=\tilde{B}$,

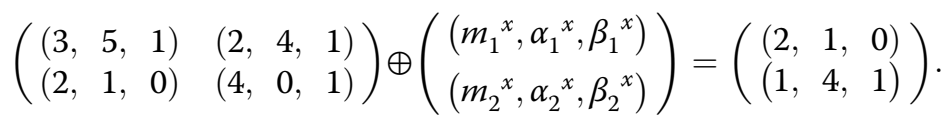

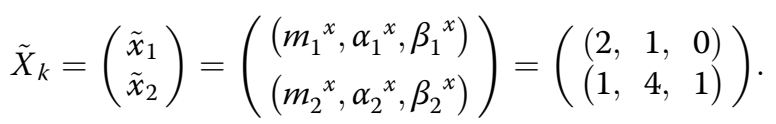

Another solution is

$$
\tilde{X}_{g}=\left(\begin{array}{c}
\tilde{x}_{1} \\
\tilde{x}_{2}
\end{array}\right)=\left(\begin{array}{c}
\left(m_{1}{ }^{x}, \alpha_{1}{ }^{x}, \beta_{1}{ }^{x}\right) \\
\left(m_{2}{ }^{x}, \alpha_{2}{ }^{x}, \beta_{2}{ }^{x}\right)
\end{array}\right)=\left(\begin{array}{l}
\left(2, \frac{51}{14}, 0\right) \\
\left(1, \frac{22}{7}, 1\right)
\end{array}\right) .
$$

We find that both fuzzy solutions satisfy the system,

$$
\tilde{A} \otimes \tilde{X}_{g}=\tilde{A} \otimes \tilde{X}_{k}=\tilde{B}
$$

Also, we can support the satisfaction of solution by the metric function because

$$
D_{2}^{2}\left(\tilde{A} \tilde{X}_{g}, \tilde{B}\right)=D_{2}^{2}\left(\tilde{A} \tilde{X}_{k}, \tilde{B}\right)=0 .
$$

The solution $\tilde{X}_{k}$ is considered as identical to solution $\tilde{X}_{k}$ '.

Hence, the FFLS may have unique solutions. In order to enhance that, an example of FFLS in size $n=3$ is illustrated with two unique solutions. 
Example 10. Consider the following $3 \times 3$ FFLS (written in the form $(m, \alpha, \beta))$.

$$
\left\{\begin{array}{c}
(3,5,1) \otimes\left(m_{1}{ }^{x}, \alpha_{1}^{x}, \beta_{1}^{x}\right) \oplus(2,4,1) \otimes\left(m_{2}{ }^{x}, \alpha_{2}{ }^{x}, \beta_{2}{ }^{x}\right) \\
\oplus(2,1,1) \otimes\left(m_{3}{ }^{x}, \alpha_{3}{ }^{x}, \beta_{3}{ }^{x}\right)=(12,24,11), \\
(2,1,0) \otimes\left(m_{1}^{x}, \alpha_{1}^{x}, \beta_{1}^{x}\right) \oplus(4,0,1) \otimes\left(m_{2}{ }^{x}, \alpha_{2}{ }^{x}, \beta_{2}{ }^{x}\right) \\
(2,3,1) \otimes\left(m_{1}{ }^{x}, \alpha_{1}{ }^{x}, \beta_{1}^{x}\right) \oplus(2,6,1) \otimes\left(m_{2}{ }^{x}, \alpha_{2}{ }^{x}, \beta_{2}{ }^{x}\right) \\
\oplus(0,0,0) \otimes\left(m_{3}{ }^{x}, \alpha_{3}{ }^{x}, \beta_{3}{ }^{x}\right)=(6,17,12),
\end{array}\right.
$$

where $\tilde{x}_{i}=\left(m_{i}^{x}, \alpha_{i}^{x}, \beta_{i}^{x}\right), i=1,2,3$ are arbitrary triangular fuzzy numbers.

Solution

The FFLS may be written in matrix form $\tilde{A} \otimes \tilde{X}=\tilde{B}$,

$$
\left(\begin{array}{lll}
(3,5,1) & (2,4,1) & (2,1,1) \\
(2,1,0) & (4,0,1) & (2,1,1) \\
(2,3,1) & (2,6,1) & (0,0,0)
\end{array}\right) \otimes\left(\begin{array}{l}
\left(m_{1}^{x}, \alpha_{1}^{x}, \beta_{1}^{x}\right) \\
\left.m_{2}^{x}, \alpha_{2}^{x}, \beta_{2}^{x}\right) \\
\left.m_{3}^{x}, \alpha_{3}^{x}, \beta_{3}^{x}\right)
\end{array}\right)=\left(\begin{array}{c}
(12,24,11) \\
(12,24,11) \\
(6,17,12)
\end{array}\right)
$$

where

$$
\tilde{X}=\left(\begin{array}{c}
\tilde{x}_{1} \\
\tilde{x}_{2} \\
\tilde{x}_{3}
\end{array}\right)=\left(\begin{array}{c}
\left(m_{1}^{x}, \alpha_{1}^{x}, \beta_{1}^{x}\right) \\
\left(m_{2}^{x}, \alpha_{2}^{x}, \beta_{2}^{x}\right) \\
\left(m_{3}^{x}, \alpha_{3}^{x}, \beta_{3}^{x}\right)
\end{array}\right) \text {. }
$$

The FFLS has two unique solutions $\tilde{X}_{1}, \tilde{X}_{2}$ as follows:

$$
\tilde{X}_{1}=\left(\begin{array}{c}
\tilde{x}_{1} \\
\tilde{x}_{2} \\
\tilde{x}_{3}
\end{array}\right)=\left(\begin{array}{c}
\left(m_{1}^{x}, \alpha_{1}^{x}, \beta_{1}^{x}\right) \\
\left(m_{2}^{x}, \alpha_{2}^{x}, \beta_{2}^{x}\right) \\
\left(m_{3}^{x}, \alpha_{3}^{x}, \beta_{3}^{x}\right)
\end{array}\right)=\left(\begin{array}{c}
\left(2, \frac{17}{10}, \frac{1}{5}\right) \\
\left(1, \frac{77}{20}, \frac{6}{5}\right) \\
\left(2, \frac{21}{20}, \frac{8}{15}\right)
\end{array}\right) .
$$

Verification for the solution:

$$
\left\{\begin{aligned}
(3,5,1) \otimes & \left(2, \frac{17}{10}, \frac{1}{5}\right) \oplus\left(1, \frac{77}{20}, \frac{6}{5}\right) \oplus(2,1,1) \otimes\left(2, \frac{21}{20}, \frac{8}{15}\right) \\
= & \left(6, \frac{52}{5}, \frac{14}{5}\right) \oplus\left(2, \frac{211}{20}, \frac{23}{5}\right) \oplus\left(4, \frac{61}{20}, \frac{18}{5}\right)=(12,24,11), \\
(2,1,0) \otimes & \left(2, \frac{17}{10}, \frac{1}{5}\right) \oplus(4,0,1) \otimes\left(1, \frac{77}{20}, \frac{6}{5}\right) \oplus(2,1,1) \otimes\left(2, \frac{21}{20}, \frac{8}{15}\right) \\
= & \left(4, \frac{37}{10}, \frac{2}{5}\right) \oplus\left(4, \frac{61}{20}, \frac{18}{5}\right)=(12,15,11), \\
(2,3,1) \otimes & \left(2, \frac{17}{10}, \frac{1}{5}\right) \oplus(2,6,1) \otimes\left(1, \frac{77}{20}, \frac{6}{5}\right) \oplus(0,0,0) \otimes\left(2, \frac{21}{20}, \frac{8}{15}\right) \\
= & \left(4, \frac{31}{5}, \frac{13}{5}\right) \oplus\left(2, \frac{54}{5}, \frac{47}{5}\right) \oplus(0,0,0)=(6,17,12) .
\end{aligned}\right.
$$


Another alternative solution is

$$
\tilde{X}_{2}=\left(\begin{array}{c}
\tilde{x}_{1} \\
\tilde{x}_{2} \\
\tilde{x}_{3}
\end{array}\right)=\left(\begin{array}{c}
\left(m_{1}^{x}, \alpha_{1}^{x}, \beta_{1}{ }^{x}\right) \\
\left(m_{2}^{x}, \alpha_{2}{ }^{x}, \beta_{2}{ }^{x}\right) \\
\left(m_{3}^{x}, \alpha_{3}^{x}, \beta_{3}{ }^{x}\right)
\end{array}\right)=\left(\begin{array}{l}
(2,1,0) \\
(1,4,1) \\
(2,1,1)
\end{array}\right) .
$$

Verification for the solution:

$$
\left\{\begin{array}{c}
(3,5,1) \otimes(2,1,0) \oplus(2,4,1) \otimes(1,4,1) \oplus(2,1,1) \otimes(2,1,1)= \\
(6,10,2) \oplus(2,11,4) \oplus(4,3,5)=(12,24,11), \\
(2,1,0) \otimes(2,1,0) \oplus(4,0,1) \otimes(1,4,1) \oplus(2,1,1) \otimes(2,1,1)= \\
(4,3,0) \oplus(4,19,6) \oplus(4,3,5)=(12,25,11), \\
(2,3,1) \otimes(2,1,0) \oplus(2,6,1) \otimes(1,4,1) \oplus(0,0,0) \otimes(2,1,1)= \\
(4,6,2) \oplus(2,11,10) \oplus(0,0,0)=(6,17,12) .
\end{array}\right.
$$

Then, the two fuzzy solutions satisfy the system

$$
\tilde{A} \otimes \tilde{X}_{1}=\tilde{A} \otimes \tilde{X}_{2}=\tilde{B} .
$$

Also, we can support satisfying the solution by distance function because

$$
D_{3}^{2}\left(\tilde{A} \tilde{X}_{1}, \tilde{B}\right)=D_{3}^{2}\left(\tilde{A} \tilde{X}_{2}, \tilde{B}\right)=0 .
$$

Now, we will transfer the example to form $(a, b, c)$ in [33],

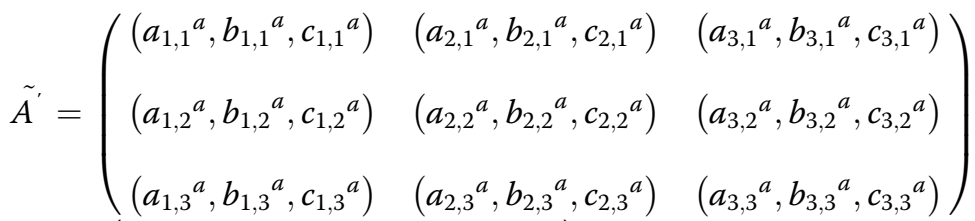

$$
\begin{aligned}
& =\left(\begin{array}{ccc}
(-2,3,4) & (-2,2,3) & (1,2,3) \\
(1,2,2) & (4,4,5) & (1,2,3) \\
(-1,2,3) & (-4,2,3) & (0,0,0)
\end{array}\right) \text {, } \\
& \tilde{X}^{\prime}=\left(\begin{array}{c}
\tilde{x}_{1} \\
\tilde{x}_{2}^{\prime} \\
\tilde{x}_{3}^{\prime}
\end{array}\right)=\left(\begin{array}{c}
\left(a_{1}^{x}, b_{1}^{x}, c_{1}^{x}\right) \\
\left(a_{2}^{x}, b_{2}{ }^{x}, c_{2}{ }^{x}\right) \\
\left(a_{3}{ }^{x}, b_{3}{ }^{x}, c_{3}{ }^{x}\right)
\end{array}\right),
\end{aligned}
$$

where $\tilde{x}_{i}{ }^{\prime}=\left(a_{i}{ }^{x}, b_{i}{ }^{x}, c_{i}{ }^{x}\right), i=1,2,3$, are arbitrary triangular fuzzy numbers. 


$$
\tilde{B}^{\prime}=\left(\begin{array}{c}
\left(a_{1}^{b}, b_{1}^{b}, c_{1}^{b}\right) \\
\left(a_{2}^{b}, b_{2}^{b}, c_{2}^{b}\right) \\
\left(a_{3}^{b}, b_{3}^{b}, c_{3}^{b}\right)
\end{array}\right)=\left(\begin{array}{c}
(12,24,11) \\
(12,25,11) \\
(6,17,12)
\end{array}\right)
$$

Then, the system may be written in the following matrix form $\tilde{A}^{\prime} \otimes \tilde{X}^{\prime}=\tilde{B}^{\prime}$

$$
\left(\begin{array}{ccc}
(-2,3,4) & (-2,2,3) & (1,2,3) \\
(1,2,2) & (4,4,5) & (1,2,3) \\
(-1,2,3) & (-4,2,3) & (0,0,0)
\end{array}\right) \otimes\left(\begin{array}{c}
\left(a_{1}^{x}, b_{1}{ }^{x}, c_{1}{ }^{x}\right) \\
\left(a_{2}{ }^{x}, b_{2}{ }^{x}, c_{2}{ }^{x}\right) \\
\left(a_{3}{ }^{x}, b_{3}{ }^{x}, c_{3}{ }^{x}\right)
\end{array}\right)=\left(\begin{array}{c}
(12,24,11) \\
(12,25,11) \\
(6,17,12)
\end{array}\right) .
$$

The first solution is

$$
\tilde{X}_{1}^{\prime}=\left(\begin{array}{c}
\tilde{x}_{1} \\
\tilde{x}_{2}^{\prime} \\
\tilde{x}_{3}^{\prime}
\end{array}\right)=\left(\begin{array}{c}
\left(a_{1}^{x}, b_{1}{ }^{x}, c_{1}{ }^{x}\right) \\
\left(a_{2}{ }^{x}, b_{2}{ }^{x}, c_{2}{ }^{x}\right) \\
\left(a_{3}{ }^{x}, b_{3}{ }^{x}, c_{3}{ }^{x}\right)
\end{array}\right)=\left(\begin{array}{c}
\left(\frac{3}{10}, 2, \frac{11}{5}\right) \\
\left(-\frac{57}{20}, 1, \frac{11}{5}\right) \\
\left(\frac{19}{20}, 2, \frac{38}{15}\right)
\end{array}\right) .
$$

The second solution is

$$
\tilde{X}_{2}^{\prime}=\left(\begin{array}{c}
\tilde{x}_{1} \\
\tilde{x}_{2}^{\prime} \\
\tilde{x}_{3}^{\prime}
\end{array}\right)=\left(\begin{array}{c}
\left(a_{1}^{x}, b_{1}{ }^{x}, c_{1}{ }^{x}\right) \\
\left(a_{2}{ }^{x}, b_{2}{ }^{x}, c_{2}{ }^{x}\right) \\
\left(a_{3}{ }^{x}, b_{3}{ }^{x}, c_{3}{ }^{x}\right)
\end{array}\right)=\left(\begin{array}{c}
(1,2,2) \\
(-3,1,2) \\
(1,2,3)
\end{array}\right) .
$$

Remark 5. The nature of uniqueness in FFLS can be determined by one solution and more, but not infinite number of solutions which is contrary to the essential concept of uniqueness on the linear system that states the determination of only one solution.

\section{Conclusion}

The nature of FFLS solution is numerically examined and revealed to be completely different from the linear programing nature of solution because the possible ways of the former solution are different from the possible ways of the linear system (no solution, unique solutions, many infinite solutions). In FFLS, the uniqueness does not happen even in the order of system $1 \times 1$. Moreover, it has been revealed that uniqueness may happen through more than one solution. The possible ways of solution do not depend upon the number of parameters compared with the number of equations, and the three former possibilities may happen even when they are equal or the number of parameters is greater or less than the number of equations.

The nature of non-fuzzy solution which is equivalent to no solution in the known possible ways of solution is recommended to be considered in the future work, and unlike the result in this paper, the LP and non-linear program (NLP) will be shown to be very effective and sufficient with no exact fuzzy solution, as only one unique approximate solution is needed in order to represent the nearest solution for FFLS; the smallest objective function is considered to be the most optimal solution. 
Received: 12 December 2013 Accepted: 23 May 2014

Published: 14 July 2014

\section{References}

1. Zadeh, LA: Fuzzy sets. Inf. Control. 8, 338-353 (1965)

2. Zadeh, LA: The concept of a linguistic variable and its application to approximate reasoning-II. Inform. Sci. 8, 301-357 (1975)

3. Allahviranloo, T, Mikaeilvand, N: Fully fuzzy linear systems solving using MOLP. World Appl. Sci. J. 12(12), 2268-2273 (2011)

4. Allahviranloo, T, Mikaeilvand, N: Non zero solutions of the fully fuzzy linear systems. Appl. Comput. Math. 10(2), 271-282 (2011)

5. Buckley, J: Solving fuzzy equations in economics and finance. Fuzzy Sets Syst. 48, 289-296 (1992)

6. Buckley, J, Qu, Y: Solving systems of linear fuzzy equations. Fuzzy Sets Syst. 43, 33-43 (1991)

7. Muzziolia, $S$, Reynaertsb, H: Fuzzy linear systems of the form $A_{1} x+b_{1}=A_{2} x+b_{2}$. Fuzzy Sets Syst. 157, 939-951 (2006)

8. Friedman, M, Ming, M, Kandel, A: Fuzzy linear systems. Fuzzy Sets Syst. 96, $201-209$ (1998)

9. Dehghan, M, Hashemi, B: Iterative solution of fuzzy linear systems. Appl. Math. Comput. 175, 645-674 (2006)

10. Kumar, A, Neetua, Bansalb, A: A new method to solve fully fuzzy linear system with trapezoidal fuzzy numbers. Can. J. Sci. Eng. Math. 1(3), 45-56 (2010)

11. Dehghan, M, Hashemi, B: Solution of the fully fuzzy linear systems using the decomposition procedure. Appl. Math. Comput. 182, 1568-1580 (2006)

12. Dehghan, M, Hashemi, B, Ghatee, M: Computational methods for solving fully fuzzy linear systems. Appl. Math. Comput. 179, 328-343 (2006)

13. Dehghan, M, Hashemi, B, Ghatee, M: Solution of the fully fuzzy linear systems using iterative techniques. Chaos Solitons Fractals 34, 316-336 (2007)

14. Dubois, D, Prade, H: Operations on fuzzy numbers. Int. J. Syst. Sci. 9(6), 613-626 (1978)

15. Abbasbandy, S, Hashemi, MS: Solving fully fuzzy linear systems by using implicit Gauss-Cholesky algorithm. Comput. Math. Model. 1, 535-541 (2012)

16. Gao, J, Zhang, QA, Gao, J, Zhang, Q: A unified iterative scheme for solving fully Fuzzy linear system. Glob. Congr. Intell. Syst. 1, 431-435 (2009)

17. Malkawi, G, Ahmad, N, Ibrahim, H: A note on "Solving fully fuzzy linear systems by using implicit Gausscholesky algorithm". Comput. Math. Model. (2014). in press

18. Mosleh, M, Otadi, M, Khanmirzaie, A: Decomposition method for solving fully fuzzy linear systems. Iran. J. Opt. 1, 188-198 (2009)

19. Nasseri, SH, Zahmatkesh, F: Huang method for solving fully Fuzzy linear system of equations. J. Math. Comput. Sci. $1(1), 1-5(2010)$

20. Nasseri, SH, Sohrabi, M, Ardil, E: Solving fully fuzzy linear systems by use of a certain decomposition of the coefficient Matrix. Int. J. Comput. Math. Sci. 3, 140-142 (2008)

21. Nasseri, S, Sohrabi, M: Cholesky decomposition for solving the fully fuzzy linear system of equations. Int. J. Appl. Math. 22(5), 689-696 (2009)

22. Nasseri, S, Matinfar, M, Kheiri, Z: Greville's method for the fully fuzzy linear system of equations. Adv. Fuzzy Sets Syst. 4, 301-3011 (2009)

23. Nasseri, S, Taleshian, F, Behmanesh, E, Sohrabi, M: A Qr-decomposition of the mean value matrix of the coefficient matrix for solving the fully fuzzy linear system. Int. J. Appl. Math. 25, 473-480 (2012)

24. Ezzati, R: Solving fuzzy linear systems. Soft. Comput. 15, 193-197 (2011)

25. Ezzati, R, Khezerloo, S, Yousefzadeh, A: Solving fully fuzzy linear system of equations in general form. J. Fuzzy Set Val. Anal. 2012, 1-11 (2012)

26. Kumar, A, Bansal, N: Solution of fully fuzzy linear system with arbitrary coefficients. Int. J. Appl. Math. Comput. 3(3), 232-237 (2011)

27. Liu, H-K: On the solution of fully fuzzy linear systems. Int. J. Comput. Math. Sci. 4(1), 29-33 (2010)

28. Kumar, A, Neetu Bansal, A: A new computational method for solving fully fuzzy linear systems of triangular fuzzy numbers. Fuzzy Inf. Eng. 4(1), 63-73 (2012)

29. Kumar, A, Babbar, N: A new computational method to solve fully fuzzy linear systems for negative coefficient matrix. Int. J. Manuf. Technol. Manag. 25, 19-23 (2012)

30. Malkawi, G, Ahmad, N, Ibrahim, H: Solving fully fuzzy linear system with the necessary and sufficient condition to have a positive solution. Appl. Math. 8(3), 1003-1019 (2014)

31. Otadi, M, Mosleh, M, Abbasbandy, S: Numerical solution of fully fuzzy linear systems by fuzzy neural network. Soft. Comput. 15, 1513-1522 (2011)

32. Babbar, $N$, Kumar, A, Bansal, A: Solving fully fuzzy linear system with arbitrary triangular fuzzy numbers $(m, a, \beta)$. Soft. Comput. 17(4), 1-12 (2012)

33. Kumar, A, Neetu, B, Kumar, A, Neetu, Bansal, A: A new approach for solving fully fuzzy linear systems. Adv. Fuzzy Syst. 2011, 1-8 (2011)

34. Malkawi, G, Ahmad, N, Ibrahim, H: A note on the nearest symmetric fuzzy solution for a symmetric fuzzy linear system. An. St. Univ. Ovidius Constanta (2014). in press

35. Dubois, D, Prade, H: Systems of linear fuzzy constraints. Fuzzy Sets Syst. 3, 37-48 (1980)

36. Kaufmann, A, Gupta, M: M: Introduction to Fuzzy. Van Nostrand Reinhold, New York, NY, USA (1991)

37. Ming, M, Friedman, M, Kandel, A: General fuzzy least squares. Fuzzy Sets Syst. 88, 107-118 (1997)

38. Otadi, M, Mosleh, M: Solving fully fuzzy matrix equations. Appl. Math. Model. 36(12), 6114-6121 (2012)

doi:10.1186/s40467-014-0016-2

Cite this article as: Malkawi et al:: On the weakness of linear programming to interpret the nature of solution of fully fuzzy linear system. Journal of Uncertainty Analysis and Applications 2014 2:16. 